\title{
Anabases
}

ANABASES Traditions et réceptions de l'Antiquité

12 | 2010

Varia

\section{Simon GOLDHILL, How to Stage Greek Tragedy Today}

\section{Malika Bastin-Hammou}

\section{OpenEdition}

Journals

Édition électronique

URL : http://journals.openedition.org/anabases/938

DOI : 10.4000/anabases.938

ISSN : 2256-9421

Éditeur

E.R.A.S.M.E.

\section{Édition imprimée}

Date de publication : 1 octobre 2010

Pagination : 251-254

ISSN : 1774-4296

\section{Référence électronique}

Malika Bastin-Hammou, "Simon goldHILL, How to Stage Greek Tragedy Today », Anabases [En ligne], 12 I 2010, mis en ligne le 01 juillet 2011, consulté le 20 septembre 2020. URL : http://

journals.openedition.org/anabases/938; DOI : https://doi.org/10.4000/anabases.938

Ce document a été généré automatiquement le 20 septembre 2020.

(c) Anabases 


\title{
Simon GOLDHILL, How to Stage Greek Tragedy Today
}

\author{
Malika Bastin-Hammou
}

\section{RÉFÉRENCE}

Simon GolDHILL, How to Stage Greek Tragedy Today, Chicago-Londres, The University of

Chicago Press, 2007, $248 \mathrm{p}$.

18 dollars / ISBN 978-0-226-30128-0.

1 Avec How to Stage Greek Tragedy Today, Simon Goldhill procure aux gens de théâtre le livre qui devrait leur permettre de mieux comprendre les tragédies grecques et de mieux les monter. L'ouvrage se donne pour objectif, en 223 pages, de cerner les grands problèmes que posent les tragédies grecques à ceux qui souhaitent les représenter et d'analyser quelques solutions données à ces problèmes par des spectacles particulièrement célèbres. Dans un style alerte et plein d'humour, l'auteur entraîne avec pédagogie le lecteur non averti dans un parcours de synthèses historiques et d'analyses précises de textes et de spectacles qui, par le point de vue adopté, sont également très stimulantes pour le spécialiste.

2 Le livre est composé d'une rapide introduction (cinq pages) suivie de six chapitres (chacun d'une quarantaine de pages), de notes, de deux pages d'indications bibliographiques et d'un index. Une vingtaine de photos en noir et blanc viennent illustrer, ça et là, le propos.

3 L'introduction rappelle les circonstances qui ont amené l'auteur à écrire ce livre et définit le projet: c'est parce qu'il n'existait pas d'ouvrage sur le théâtre antique spécifiquement destiné aux acteurs et metteurs en scène que ce livre a été écrit. Face à l'engouement des metteurs en scène pour les tragédies grecques que l'on constate depuis une trentaine d'années, et parce que certaines mises en scène ont eu un succès exceptionnel, l'auteur se propose d'aider les nombreux metteurs en scène qui envisagent de monter une tragédie grecque en s'appuyant sur l'analyse de quelques 
spectacles particulièrement convaincants. Il ne s'agit donc ni d'un ouvrage érudit sur le théâtre antique, ni d'un travail exhaustif sur la réception du théâtre antique, non plus que d'un manuel à l'usage des metteurs en scène - mais c'est un peu les trois à la fois. La méthode est en effet pragmatique : partant des grands problèmes que posent les tragédies grecques en général, l'auteur expose ce que l'on sait des données antiques relatives à ces problèmes, puis examine la façon dont les spectacles y ont répondu et analyse alors le détail des textes en tâchant d'en révéler les lignes de force. Il ne s'agit donc pas du tout d'être prescriptif, encore moins d'inciter à des mises en scène «à l'antique ", mais de comprendre pourquoi «les pièces antiques sont si difficiles à monter bien » (p. 3).

4 Le premier chapitre porte sur l'espace théâtral. Partant des spécificités de l'espace théâtral antique, l'auteur analyse ce qu'elles impliquent et comment les modernes peuvent en tenir compte. L'opposition entre l'orchestra et le proskénion, l'importance de la porte centrale de la skénè, les parodoi, qui permettent de longues entrées et sorties des acteurs, ou encore la hauteur de la skénè, qui crée un axe vertical, constituent la matrice spatiale de la tragédie. Rares sont les théâtres modernes qui offrent toutes ces potentialités, mais peu importe : pour l'auteur, l'essentiel est de créer «a theatrical space that can recognize and work with the way that Greek tragedy has been written " (p.11). Il analyse alors comment Guy-Claude François, le scénographe d'Ariane Mnouchkine, a remarquablement bien réinterprété les données de l'espace scénique antique en 1990 pour la création des Atrides, notamment en donnant toute sa place au chœur. À l'inverse, la Médée de Deborah Warner, en 2001, parce qu'elle ne réinterprétait pas l'axe vertical, omettait la dimension divine de la pièce - Médée ne s'envolait plus sur un char - et réduisait la tragédie à la sphère bourgeoise et domestique. De fait, chaque tragédie a sa propre symbolique de l'espace, qui vient s'ajouter aux données spatiales des théâtres antiques. L'auteur le montre rapidement en proposant une analyse comparée des Choéphores d'Eschyle et d'Électre de Sophocle : un même mythe, un même espace théâtral donnent lieu à deux dramaturgies radicalement différentes. Chez Eschyle, la tombe d'Agamemnon est au centre de l'espace et entre en tension avec la porte du palais, d'où le roi et son fils ont été exclus. Chez Sophocle c'est Électre qui occupe, d'un bout à l'autre de la pièce, l'espace scénique, qui est un espace vide dans lequel l'héroïne tourne en rond et parle quand elle devrait se taire, à l'intérieur du palais.

5 Le deuxième chapitre est consacré au chœur tragique. C'est en effet l'élément le plus étranger aux spectateurs modernes et un véritable cauchemar pour qui veut éviter l'effet « comédie musicale » débitant des banalités sans fin sur le sens de la vie. L'auteur distingue trois fonctions du chœur : il incarne les valeurs religieuses et sociales de la communauté ; il fournit un commentaire à l'action en la confrontant aux mythes et à la sagesse populaire qui sont la culture partagée des spectateurs; il devient ainsi une figure d'autorité. Mais, parce que les personnages qui composent le chœur sont bien souvent des figures marginalisées - esclaves, femmes, étrangers, vieillards - et exclues des lieux d'autorité du monde grec, le chœur en vient à combiner des facettes contradictoires qui rendent son interprétation particulièrement délicate. Il n'est donc pas aisé de trouver des équivalents à la collectivité que représente le chœur. Plusieurs solutions ont été proposées par les metteurs en scène : Gospel at Colonus faisait du chœur des vieillards d'Edipe roi un chœur de gospel; Ariane Mnouchkine a puisé son inspiration du côté de théâtres traditionnels indiens comme le kathakali, le bharata natyam et le kûtiyattam; d'autres encore se sont tournés vers les fanfares des Balkans - 
autant de solutions qui permettent d'échapper au réalisme politique que suscitent souvent les tragédies grecques.

6 Le troisième chapitre est consacré aux acteurs. L'auteur examine les implications des conventions régissant les textes dramatiques antiques, et notamment le récit de messager. Il est étudié en détail, au-delà du discours convenu qui le réduit au récit d'événements impossibles à montrer sur scène, par un personnage qui n'a pas d'autre fonction dans la pièce. L'auteur montre que certaines tragédies multiplient les personnages de messagers pour mieux les opposer - c'est le cas d'EEdipe roi; dans d'autres, ce sont les personnages qui deviennent, temporairement, des messagers. Ainsi dans Électre, c'est successivement le précepteur d'Oreste puis Électre qui endossent le rôle, le premier se faisant metteur en scène de la ruse qu'il a inventée pour rendre le trône à Oreste, tandis que la seconde commente et encourage le meurtre de sa propre mère. Les messagers sont alors fortement impliqués dans les scènes dont ils font le récit. La difficulté majeure pour les comédiens d'aujourd'hui reste selon l'auteur de résister au réflexe d'une interprétation psychologique des personnages antiques, qui sont des types plus que des individus. L'auteur analyse les solutions proposées par Ariane Mnouchkine et Peter Hall : tous deux ont fait le choix d'un jeu stylisé, codifié, débarrassé de tout réalisme psychologique.

7 Dans le quatrième chapitre, l'auteur s'intéresse à ce qui provoque, chez les metteurs en scène contemporains, l'envie de monter des tragédies grecques : leur portée politique. De fait, comme il le rappelle à juste titre, la tragédie grecque est intrinsèquement liée au cadre civique qui l'a vue naître, en même temps qu'elle en constitue la critique. Civique et critique, la tragédie est un genre qui pose les problèmes auxquels est confrontée la cité et leur fait face. Mais pour ce faire, elle emprunte un chemin détourné qui est celui de l'altérité. Contrairement à la comédie, la tragédie se passe ailleurs et autrefois. Elle met en scène les "autres" - étrangers, femmes, esclaves, vieillards : «Tragedy raises questions about each of us... yet to do so it takes a detour through the other. [p. 138]» L'auteur rapporte plusieurs expériences de représentations d'Électre en Irlande, et leur grande efficacité auprès de spectateurs qui auraient peut-être rejeté une pièce s'adressant plus directement à eux. Il met en garde les metteurs en scène qui seraient tentés, en recourant aux tragédies grecques, par le didactisme, l'envie de "faire passer un message » : la tragédie grecque pose beaucoup de questions politiques, mais ne donne pas de réponses. Ainsi, les victimes pour lesquelles le public éprouve de la compassion se révèlent souvent d'affreux bourreaux que l'on pense à la Médée d'Euripide... C'est, après Antigone, la tragédie grecque la plus montée, sans doute parce que comme Antigone elle pose la question du genre. Les femmes, rappelle l'auteur, sont très présentes dans les tragédies grecques, ces spectacles composés et joués par des hommes, pour des hommes. Souvent victimes, on les voit œuvrer pour préserver la maisonnée de la destruction masculine. Et en même temps, la rhétorique misogyne y est omniprésente, précisément parce que la définition du masculin est mise en danger par la tragédie : « Masculinity's self-assertion becomes a violent and self-destructive spectacle. [p. 150]» La tragédie procède donc par un détour qui permet de révéler les failles de ceux qui y assistent, qu'il soient des Grecs du $\mathrm{v}^{\mathrm{e}} \mathrm{s}$. avant notre ère ou nos contemporains.

8 Le cinquième chapitre pose la question de la traduction. Écartant d'emblée les textes à visée universitaire, l'auteur envisage ceux composés spécifiquement pour la scène et qu'on aurait plutôt tendance, en France, à qualifier d'adaptations. Il limite d'ailleurs 
son propos aux traductions en langue anglaise, ce qui rend ce chapitre moins intéressant pour les francophones, même si les conclusions générales peuvent s'appliquer aux traductions françaises. Il oppose ainsi les traductions qui font le choix de la clarté, au risque de l'« oversimplification", à celles préférant rester obscures quand le grec l'est aussi, concluant que, à la question " What is the best translation? ", la meilleure réponse est sans doute « For what type of performance?» (p. 162), et que l'idéal serait d'élaborer une nouvelle traduction pour chaque nouveau spectacle, comme pour les décors ou les costumes.

Enfin, le sixième et dernier chapitre est consacré aux personnages du théâtre antique qui posent des problèmes de représentation aux metteurs en scène et spectateurs d'aujourd'hui : fantômes, dieux, héros... et Hélène de Troie. Au point que les tragédies dans lesquelles on trouve les uns ou les autres sont souvent délaissées au profit de celles qui font moins courir le risque de sombrer dans le grotesque. Sans proposer de solution, l'auteur met ici en garde contre les facilités et se livre à un savoureux bêtisier - rejetant notamment l'équivalence entre Hélène de Troie, dont il rappelle qu'aucun texte antique ne décrit sa beauté, et Marilyn Monroe...

$10 \mathrm{Au}$ final, cet ouvrage est très stimulant et agréable à lire. Les antiquisants le parcourront avec plaisir, même si l'on peut trouver dommage la limitation du champ d'investigation au domaine anglophone, aussi bien pour les mises en scène que pour les traductions et la bibliographie: on regrettera ainsi particulièrement l'absence de référence au très bon livre, assez proche et plus ancien, de Patricia Legangneux, $L a$ tragédie grecque sur la scène moderne. Une utopie théâtrale. Ce sont néanmoins les gens de théâtre qui en tireront le plus grand profit - et à ce titre, une traduction française serait tout à fait bienvenue.

\section{AUTEURS}

\section{MALIKA BASTIN-HAMMOU}

Université de Grenoble

Malika.Bastin@u-grenoble3.fr 